\title{
Bullous and hemorrhagic lichen sclerosus - Case report *
}

\author{
Raquel Sucupira Andrade Lima ${ }^{1}$ \\ Antônio Pedro Mendes Schettini ${ }^{1}$
}

\author{
Gustavo Ávila Maquiné ${ }^{1}$ \\ Mônica Santos ${ }^{1,2}$
}

DOI: http:/ / dx.doi.org/10.1590/abd1806-4841.20153502

\begin{abstract}
A bstract: Lichen sclerosus is a chronic inflammatory disease, usually located in the genital area. The etiology of lichen sclerosus is multifactorial, with participation of genetic, autoimmune, infectious and hormonal factors. Bullous clinical form stems from hydropic degeneration of the basal membrane, constituting a less frequent variant of the disease. In this work, we report the case of a female patient, 55 years old, who in the last three years presented whitish plaques, with horny spikes, located on back and arms. Some of these lesions evolved with hemorrhagic blisters, which after histopathological examination confirmed the diagnosis of bullous and hemorrhagic lichen sclerosus. The patient was treated with high-potency topical corticosteroid for two months, resulting in remission of bullous and hemorrhagic lesions.
\end{abstract}

Keywords: Clinical diagnosis; Lichen sclerosus et atrophicus; Primary treatment

\section{INIRODUCTION}

Lichen sclerosus is a rare inflammatory mucocutaneous dermatosis, clinically described for the first time by Hallopeau in 1887 , with its histopathological characteristics defined by Darier in $1892 .{ }^{1}$ It can affect both sexes and all age groups; however, it is prevalent in females (rate of 10 women for each man affected), mainly between the $5^{\text {th }}$ and $6^{\text {th }}$ decades of life. ${ }^{2}$ It occurs predominantly in the genital area, with extragenital lesions in 15 to $20 \%$ of the patients. Clinically, extragenital lesions are characterized by the presence of papules and erythematous plaques which become progressively whitish and hardened, with superficial horny spikes. They are usually asymptomatic, although pruritus may occur in variable degrees. ${ }^{3}$ In this study, we report the case of a patient with bullous and hemorrhagic lichen sclerosus, atypical and rarer variant of the disease.

\section{CASE REPORT}

Female patient, 55 years old, dental assistant, evolved with asymptomatic lesions on the back and upper limbs for three years. At the dermatological examination, whitish plaques of hardened consistency, with well-defined borders and horny spikes were observed on the back and upper limbs. There were also plaques with hemorrhagic aspect, with superficial horny spikes and blisters, located on the back (Figure 1). During this time of evolution, she underwent two cutaneous biopsies at previous evaluations carried out at other outpatient clinics, with histological diagnosis suggesting circumscribed lymphangioma and scleroderma plaque, respectively. She was referred to a REFERENCES center, undergoing a new biopsy; the histopathological examination revealed atrophic epidermis, with hyperkeratosis and follicular plugs. Vacuolar degeneration of the basal layer was observed 
in the dermis, with subepidermal blisters and extravasation of red blood cells. Edema and collagen homogenization in papillary dermis were also observed, with a perivascular lymphohistiocytic inflammatory infiltrate, confirming the diagnosis of bullous and hemorrhagic lichen sclerosus (Figure 2). Routine laboratory tests did not show other changes. The patient was treated with high-potency topical corticoid, evolving to remission of the hemorrhagic bullous lesions after 2 months of treatment. She remains under clinical follow-up, with hyperkeratotic lesions on the back (Figure 3 ).

\section{DISAUSSION}

The etiology of lichen sclerosus has not yet been clarified, but studies demonstrate the involvement of genetic, hormonal and autoimmune factors. The participation of infectious agents (such as infection by Borrelia burgdorferi, papillomavirus and Hep- atitis $C$ virus) and trauma has also been implicated in triggering the disease. ${ }^{4,5}$ The most common location is the genital area. The extragenital form occurs in approximately $20 \%$ of the patients, and in the majority of these cases there are concomitant genital lesions. The isolated extragenital lichen sclerosus is rarer, and may have an unusual clinical presentation, like the development of vesiculobullous lesions. In these cases, the lesions involve preferably the trunk, neck and proximal extremities of arms, are usually asymptomatic and without evidences of malign transformation. ${ }^{6}$ Bullous LE may be localized or generalized, and has already been described with distribution according to Blaschko lines. Forms with hemorrhagic blisters are less frequent. ${ }^{7}$ The mechanism through which these blisters are formed has not been totally elucidated yet, but it is believed that it occurs by intensive hydropic degeneration of the basal layer, with fragility of dermal-epidermal junction and formation of sub-
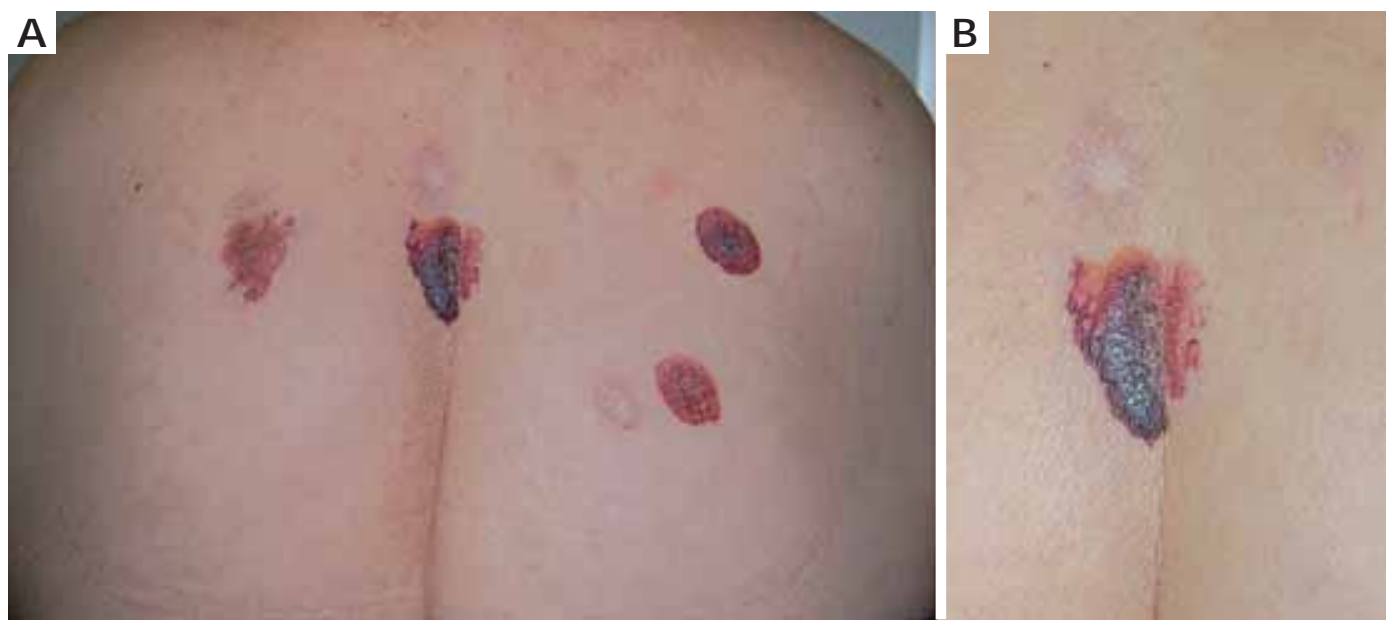

\section{FIGURE 1:}

Whitish and sclerotic plaques on the back, some with hemorrhagic aspect, horny spikes and superficial blisters (A). Detail of plaque with superficial hemorrhagic blisters and horny spikes (B)
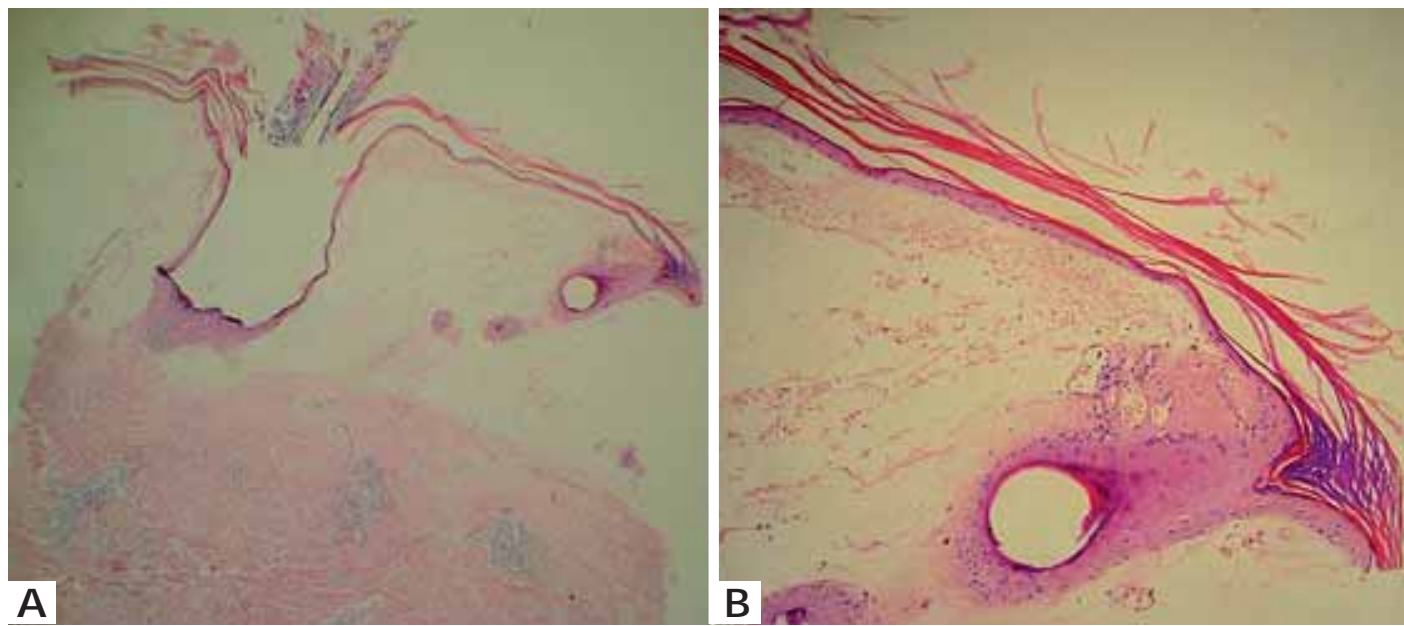

FIGURE 2:

Histological examination, with hematoxilin-eosin staining, revealed atrophic epidermis, with hyperkeratosis and follicular plugs. In the dermis, vacuolar degeneration of basal layer was observed, with subepidermal blister and extravasation of red blood cells 

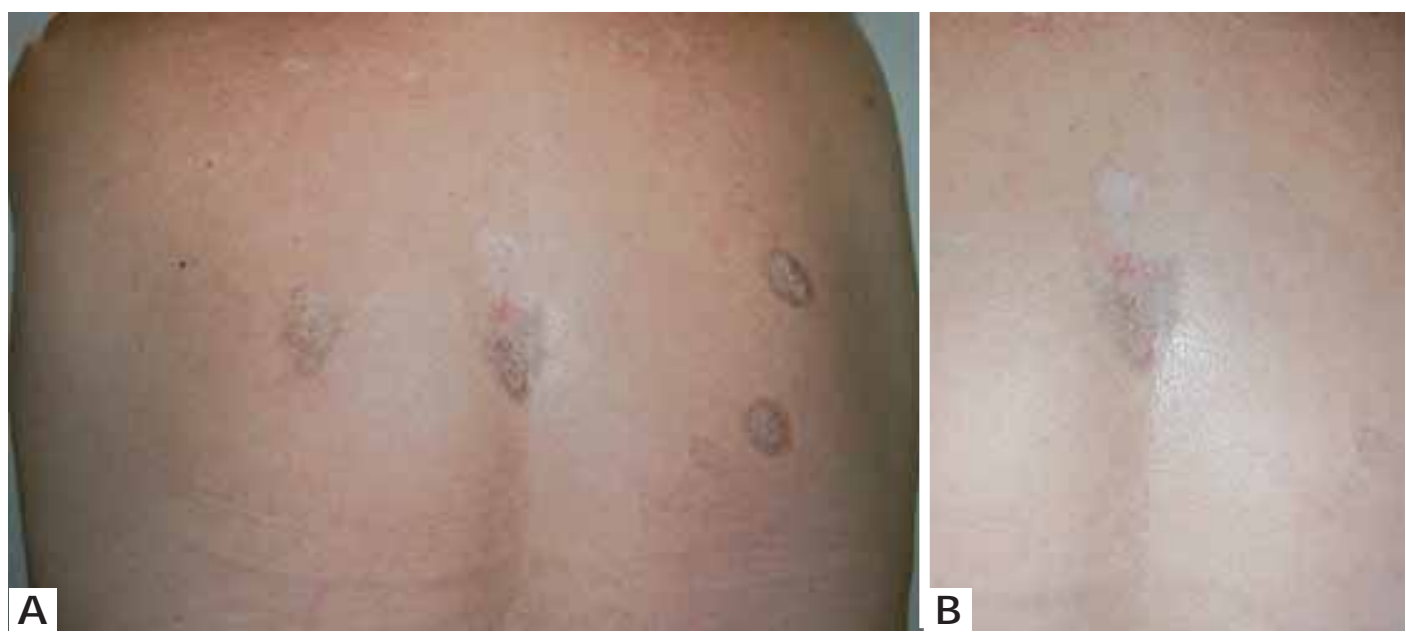

\section{Figure 3:}

After treatment with high-potency topical corticoids for 2 months, there was remission of the hemorrhagic bullous lesions, with some hyperkeratotic lesions persisting on the back

epidermal blisters. ${ }^{8}$ Differential diagnosis of the bullous variant of LE includes cicatricial bullous pemphigoid, bullous scleroderma, bullous lichen planus and circumscribed lymphangioma. ${ }^{9}$ As for the treatment, several therapeutic modalities are reported, such as topical corticosteroids, retinoids, tacrolimus and tes- tosterone, besides UVA phototherapy, isolated or combined with topical drugs. Some authors further report that these bullous lesions may involute leaving hyperkeratotic plaques typical of the disease, which are normally more refractory to the usual therapies, similarly to what occurred in the reported case. ${ }^{10}$

\section{REFERENCES}

1. Fistarol SK, Itin PH. Diagnosis and Treatment of Lichen Sclerosus- An Update. Am J Clin Dermatol. 2013;14:27-47.

2. Rose AE, Boyd KP, Meehan SA, Latkowski JA. Lichen sclerosus et atrophicus. Dermatol Online J. 2013;19:20714.

3. Zendell K, Edwards L. Lichen sclerosus with vaginal involvement: report of 2 cases and review of the literature. JAMA Dermatol. 2013;149:1199-202.

4. Santos M, Haddad Júnior V, Ribeiro-Rodrigues R, Talhari S. Lyme borreliosis. An Bras Dermatol. 2010:85:930-8.

5. Bjekić M, Šipetić S, Marinković J. Risk factors for genital lichen sclerosus in men. Br J Dermatol. 2011:164:325-9.

6. Lavorato FG, Nery NS, Bomm L, Mann D, Gripp AC, Alves Mde F. Cases for diagnosis. An Bras Dermatol. 2013;88:297-8

7. Kimura A, Kambe N, Satoh T, Togawa Y, Suehiro K, Matsue H. Follicular keratosis and bullous formation are typical signs of extragenital lichen sclerosus. J Dermatol. 2011;38:834-6.

8. Rotsztejn H, Trznadel-Grodzka E, Krawczyk T. Lichen sclerosus mimicking Bowen's disease. Postep Derm Alergol. 2012;4:321-3.

9. Ballester I, Bañuls J, Pérez-Crespo M, Lucas A. Extragenital bullous lichen sclerosus atrophicus. Dermatol Online J. 2009;15:6.

10. Khatu S, Vasani R. Isolated, Localised Extragenital Bullous Lichen Sclerosus et Atrophicus: A Rare Entity. Indian J Dermatol. 2013;58:409.

\author{
M AILING ADDRESS: \\ M ônica Santos \\ A v. Codajas, 25 \\ Cachoeirinha \\ 69065-130 - M anaus - A M \\ Brazil \\ E-mail: m.n.souza.santos@gmail.com
}

How to cite this article: Lima RSA, Maquiné GA, Schettini APM, Santos M. Bullous and hemorrhagic lichen sclerosus - case report. An Bras Dermatol. 2015;90 (3 Suppl 1):S118-20. 